Research Article

\title{
Optimal Hedging and Pricing of Equity-LinkedLife Insurance Contracts in a Discrete-Time Incomplete Market
}

\author{
Norman Josephy, Lucia Kimball, and Victoria Steblovskaya \\ Department of Mathematical Sciences, Bentley University, 175 Forest Street, Waltham, \\ MA 02452-4705, USA \\ Correspondence should be addressed to Lucia Kimball, 1kimball@bentley.edu
}

Received 26 May 2011; Accepted 1 September 2011

Academic Editor: Tomasz J. Kozubowski

Copyright (C) 2011 Norman Josephy et al. This is an open access article distributed under the Creative Commons Attribution License, which permits unrestricted use, distribution, and reproduction in any medium, provided the original work is properly cited.

\begin{abstract}
We present a method of optimal hedging and pricing of equity-linked life insurance products in an incomplete discrete-time financial market. A pure endowment life insurance contract with guarantee is used as an example. The financial market incompleteness is caused by the assumption that the underlying risky asset price ratios are distributed in a compact interval, generalizing the assumptions of multinomial incomplete market models. For a range of initial hedging capitals for the embedded financial option, we numerically solve an optimal hedging problem and determine a risk-return profile of each optimal non-self-financing hedging strategy. The fair price of the insurance contract is determined according to the insurer's risk-return preferences. Illustrative numerical results of testing our algorithm on hypothetical insurance contracts are documented. A discussion and a test of a hedging strategy recalibration technique for long-term contracts are presented.
\end{abstract}

\section{Introduction}

Equity-linked life insurance provides the insured with the opportunity of participating in the growth potential of an equity-based financial market index such as the S\&P 500 index. In addition, equity-linked insurance with guarantee provides downside protection with a guaranteed minimum return. The combination of equity participation and downside protection significantly improves the desirability of the insurance contract.

From the perspective of the insurance issuer, such insurance contracts create two sources of risk. One source of risk is mortality risk. Mortality risk is related to the likelihood of an insurance-type event such as client's death prior to contract maturity or client's survival to contract maturity. The equity-linked component of the insurance contract, which is related 
to the behavior of the underlying risky asset, creates the second source of risk, a financial risk. A fair price of an equity-linked life insurance contract should account for both sources of risk.

The pricing and hedging of equity-linked life insurance contracts are an active area of research. In their pioneering work, Brennan and Schwartz [1,2] provided an initial impetus to combining actuarial and financial risk management approaches by showing that the payable benefit for equity-linked insurance contract could be viewed as a known guarantee amount and the pay-off of an embedded call option. This approach has been developed by many authors, see, for example, [3,4]. These authors develop their models under the major assumption of financial market completeness.

Several authors consider an incomplete insurance market, where incompleteness is caused by mortality risk, an additional risk factor independent of the financial risk, while the financial market itself is described by a complete market model. In [5], the author applies risk-minimization approach developed in $[6,7]$ to determine non-self-financing hedging strategies for equity-linked pure endowment contracts. The financial market itself is described by the complete discrete binomial model.

In a series of papers by Melnikov et al. [8-10], the methods of quantile hedging as well as efficient hedging developed in [11], [12], respectively, are applied to pricing and hedging of various unit-linked and equity-linked life insurance contracts. Here the financial market itself is described by the complete single- or multidimensional Black-Scholes model or by a jump-diffusion model with the unique equivalent risk-neutral measure. Klusik and Palmowski in their recent work [13] rely on methods of quantile hedging as well as on their original results to find optimal self-financing hedging strategies for more complex payoffs in the framework of the Black-Scholes financial market with reduced initial hedging capital.

Incomplete financial markets in the context of equity-linked life insurance have been studied in [14]. Here financial market incompleteness is caused by the dynamics of the underlying risky asset which follows a continuous time stochastic process with jumps. In [15], a stochastic programming model is developed to account for various sources of financial market incompleteness, such as jumps in the underlying asset stochastic process and heteroscedasticity of the process for the underlying asset.

In the present paper, we consider an equity-linked life insurance contract in the framework of an incomplete discrete-time financial market originally developed in [16]. The market incompleteness is caused by the behavior of the underlying risky asset. The risky asset price ratios at every time point are assumed to be distributed over a bounded interval, in contrast to a complete binomial model where they have a two-point distribution. This market model is a natural extension of the incomplete multinomial market models previously developed in the literature (see, e.g., [17]). In [18-20], an algorithmic approach to optimal hedging of various contingent claims (path independent as well as path dependent) was developed. The algorithm uses an available initial hedging capital to produce a non-selffinancing hedging strategy which is optimal with respect to a chosen risk criterion. This approach presents a more flexible alternative to other methods for hedging in incomplete markets, such as local risk minimization (see, e.g., [21] and the references therein). In [22] comparative numerical results that illustrate better performance of our alternative strategies compared to local quadratic and local linear risk minimization strategies are presented.

We further extend our previous work to develop a technique for both optimal hedging and pricing of equity-linked life insurance products in an incomplete discrete-time financial market environment. In this paper, we use a pure endowment contract with guarantee as an example to illustrate our methodology. Our method is extensible to more sophisticated equity-linked products. 
In our approach, the pricing of a pure endowment contract with guarantee begins with determining a range of admissible initial hedging capital values. For each of the chosen initial capital values, we identify a non-self-financing hedging strategy which optimizes an insurer relevant risk criterion and determine a risk-return profile of the optimal strategy.

The insurance company chooses the risk-return profile that agrees with their preferences. Based on the initial hedging capital associated with the preferred risk-return profile and using the customer's survival probability, the insurer determines the fair price of the contract.

Our numerical algorithm is applied to two hypothetical pure endowment contracts with guarantee where the underlying risky asset is the S\&P 500 index.

As an illustration of our algorithm flexibility, we show how our optimal hedging strategy can be recalibrated during the lifetime of a long-term contract in order to take into account the most recent market data. Numerical simulations show that a recalibrated hedging strategy has improved risk-return characteristics.

The remainder of this paper is organized as follows. In Section 2, we set up a problem of pricing and optimal hedging of an equity-linked pure endowment insurance contract and relate it to the problem of optimal hedging in an incomplete market. A detailed development of our incomplete market model and our algorithmic approach to optimal hedging is presented in Section 3. We discuss application of our approach to equity-linked life insurance products in Section 4. Numerical results of application of our algorithm to two pure endowment life insurance contracts with guarantee are presented in Section 5. Section 6 is devoted to a discussion and a test of our recalibration technique.

\section{Problem Setting}

Let $\left(\Omega, F, D, F_{i}\right)$ denote a filtered probability space, where $\Omega$ is a sample space, $D$ is a probability measure on the $\sigma$-algebra $F$ of subsets of $\Omega$, and $\left(F_{i}\right)_{i=0,1, \ldots, n}$ is a filtration, an increasing sequence of $\sigma$-algebras $F_{0} \subseteq F_{1} \subseteq \cdots \subseteq F_{n}$. Here $F_{i}$ corresponds to the discretetime moment $t_{i}, 0=t_{0}<t_{1}<\cdots<t_{n}=T(T>0), F_{0}=\{\emptyset, \Omega\}$, and $F_{n}=F$.

Let $S=\left(S_{i}\right)_{i=0,1, \ldots, n}$ denote a discrete-time strictly positive risky asset price process, adapted to the filtration $\left(F_{i}\right)_{i=0,1, \ldots, n}\left(S_{i}\right.$ is $F_{i}$-measurable for all $\left.0 \leq i \leq n\right)$. Let $B_{i}=$ $B_{0}(1+r)^{i}, B_{0}>0$ be a deterministic riskless asset (bond) process with constant interest rate $r$. Without loss of generality we will assume that $B_{0}=1$.

We consider a financial market consisting of the risky asset and bond that can be traded at times $0=t_{0}<t_{1}<\cdots<t_{n}=T(T>0)$. Suppose the following additional assumption holds.

(A1) The risky asset price ratios (jumps) $\psi_{i}=S_{i} / S_{i-1}$ are distributed over a bounded interval $[D, U]$ for all $1 \leq i \leq n$, where $D<1+r<U$.

No further assumptions are made regarding the risky asset price jump distribution.

This discrete-time market model is incomplete. In fact, it represents a natural extension of the incomplete multinomial discrete-time model (see, e.g., [17]) which in turn generalizes the famous complete binomial model [23]. More details about our market model will be given in Section 3.

Let us denote by $H$ a European-type contingent claim on the underlying asset $S$, with maturity $T=t_{n}$ (meaning that $H$ is a $F_{n}$-measurable random variable).

By $T(x)$ we denote the remaining lifetime of a person who is currently $x$ years old. We assume that $T(x)$ is a random variable defined on a probability space $(\widetilde{\Omega}, \widetilde{F}, \widetilde{p})$. 
Traditionally, it is assumed that financial and mortality factors are independent (see, e.g., [24]). We will adopt this point of view as well. Hence, we will assume that the probability spaces $(\Omega, F, D)$ and $(\widetilde{\Omega}, \widetilde{F}, \widetilde{p})$ are independent.

We consider a single-premium equity-linked life insurance contract where the insured person receives the amount $H$ provided that he/she is alive at maturity $T$ (a pure endowment equity-linked life insurance contract). The pay-off (benefit) at maturity of such insurance contract is as follows:

$$
\widehat{H}=H \cdot I_{\{T(x)>T\}} .
$$

Here $I_{\{T(x)>T\}}$ is the indicator function of the event $\{T(x)>T\}$. This function takes the value of 1 if the insured person survives beyond the contract maturity and 0 in the opposite case.

We will take the position of an insurance company that issues a pure endowment life insurance contract and needs to determine a fair price of such contract. In addition to determining an appropriate contract price, an insurance company is looking to find an optimal way to invest in a hedging strategy to protect their position in the contract in accordance with their risk preferences.

Since our financial market is incomplete, there exists an infinite set of equivalent riskneutral probability measures on $(\Omega, F)$. Let $p^{*}$ be some equivalent risk-neutral measure; that is, $D^{*}$ is a probability measure, which is equivalent to $D$ and such that the discounted risky asset price process $Z=\left(Z_{i}\right)_{i=0,1, \ldots, n}, Z_{i}=S_{i} / B_{i}$ is a $p^{*}$-martingale.

Then the time-zero no-arbitrage price ${ }_{T} U_{x}$ of the life insurance contract associated with $p^{*}$ is

$$
\begin{aligned}
{ }_{T} U_{x} & =\mathcal{E}^{*} \times \tilde{\mathcal{E}}\left(\widehat{H}(1+r)^{-n}\right) \\
& =\mathcal{E}^{*} \times \tilde{\mathcal{E}}\left(H(1+r)^{-n} \cdot I_{\{T(x)>T\}}\right),
\end{aligned}
$$

where $\mathcal{E}^{*} \times \tilde{\mathcal{E}}$ denotes the expectation with respect to the product measure $p^{*} \times \tilde{p}$. Further, using the independence of $p^{*}$ and $\tilde{p}$, we obtain

$$
{ }_{T} U_{x}=\mathcal{E}^{*}\left(H(1+r)^{-n}\right){ }_{T} p_{x}
$$

where ${ }_{T} p_{x}=\tilde{p}(T(x)>T)$ is the survival probability; that is, the probability that an insured person of age $x$ survives beyond time $T$.

In the case of a complete financial market, the measure $D^{*}$ is unique, and therefore the quantity $\mathcal{E}^{*}\left(H(1+r)^{-n}\right)$ occurring in (2.3) is a unique no-arbitrage price of the contingent claim $H$. This quantity represents a perfect initial hedging capital that can be used to construct a self-financing hedging strategy (based on risky assets and bonds) which perfectly replicates the contingent claim $H$. However, the presence of a mortality factor makes the market incomplete, since only the reduced quantity $\mathcal{E}^{*}\left(H(1+r)^{-n}\right)_{T} p_{x}$ is available to the hedger. There are a variety of approaches (see, e.g., $[13,25]$ and the references therein) that build optimal self-financing hedging strategies based on the reduced initial capital in the framework of a complete financial market.

In our setting, the financial market under consideration is incomplete. Market incompleteness results in the fact that the measure $D^{*}$ is not unique and therefore the quantity 
$\mathcal{E}^{*}\left(H(1+r)^{-n}\right)$ is no longer a unique no-arbitrage price of the contingent claim $H$. A perfect hedge with a self-financing strategy is not possible even if the mortality factor is not present. In our approach to hedging, we consider non-self-financing hedging strategies (based on risky assets and bonds) that are funded by the available initial hedging capital. We introduce optimization criteria meaningful to the investor and numerically solve the appropriate optimization problems. Our approach to pricing is based on the analysis of the risk-return characteristics of the optimal hedging strategies.

\section{Pricing and Hedging of Contingent Claims in a Discrete-Time Incomplete Market}

In this section, we give an overview of the discrete-time incomplete market model and our algorithmic approach to optimal hedging of contingent claims (see [18-20]). We will return to optimal hedging and pricing of equity-linked life insurance contracts in Section 4.

\subsection{No-Arbitrage Prices}

Let us consider the financial market model introduced in Section 2 under assumption (A1). Additionally, we will assume that the following holds.

(A2) A European contingent claim $H$ has a convex continuous pay-off function $f$.

In our incomplete financial market model, there exists an infinite set of equivalent riskneutral measures on $(\Omega, F)$. At each time $t_{i}$, each equivalent risk-neutral measure $D^{*}$ produces the no-arbitrage price $\mathcal{E}^{*}\left(H(1+r)^{-(n-i)}\right)$ for a European contingent claim $H$ with the pay-off function $f$. These no-arbitrage contingent claim prices at each time $t_{i}$ form an open interval:

$$
\left(\underline{Y}_{i}(f, D, U), \bar{Y}_{i}(f, D, U)\right), \quad i=0, \ldots, n-1,
$$

where parameters $D$ and $U$ determine the support of the risky asset price ratio distribution (see assumption (A1)). The explicit formulas for the upper and lower bounds of the noarbitrage price interval for the case where $H$ is a European call option were first obtained in [26]. Further, these formulas have been generalized to the case of a European option with a convex pay-off function in [27], see also [16, 17, 28-30].

We will need the following definition.

Definition 3.1. Consider the financial market described in Section 2. Replace assumption (A1) with the framework of the Cox-Ross-Rubinstein (CRR) complete financial market binomial model with parameters $\mathfrak{D}$ and $\mathfrak{U}(\mathfrak{D}<1+r<\mathfrak{U})$ (i.e., the risky asset price ratio $\psi_{i}=S_{i} / S_{i-1}$ at any time $t_{i}(i=1, \ldots, n)$ takes one of the two possible values: $\mathfrak{D}$ or $\left.\mathfrak{U}\right)$. Suppose the contingent claim $H$ with the pay-off function $f$ and the initial risky asset price $S_{0}$ is evaluated in the framework of the above CRR model. One will define $\operatorname{CRR}_{i}(f, \mathfrak{D}, \mathfrak{U})$ as the unique CRR contingent claim price at time $t_{i}$.

Example 3.2. Suppose $H$ is a European type path-independent option with the pay-off function $f$ that depends only on the terminal risky asset value. Then in the framework of the Cox-Ross-Rubinstein binomial model, we have

$$
\mathrm{CRR}_{i}(f, \mathfrak{D}, \mathfrak{U})=g_{i}\left(\mathfrak{D}, \mathfrak{U}, S_{i}\right),
$$


where

$$
\begin{gathered}
g_{i}(\mathfrak{D}, \mathfrak{U}, x)=(1+r)^{-(n-i)} \sum_{j=0}^{n-i}\left(\begin{array}{c}
n-i \\
j
\end{array}\right) P^{j}(1-P)^{n-i-j} f\left(x \mathfrak{U}^{j} \mathfrak{D}^{n-i-j}\right), \\
P=\frac{(1+r)-\mathfrak{D}}{\mathfrak{U}-\mathfrak{D}} .
\end{gathered}
$$

We have the following proposition.

Proposition 3.3. Let $H$ be a European contingent claim with the pay-off function $f$. Suppose assumptions (A1) and (A2) hold.

Then the upper bound at time $t_{i}$ of the no-arbitrage price interval for $\mathrm{H}$ is given by the following formula:

$$
\bar{Y}_{i}(f, D, U)=C R R_{i}(f, D, U) \quad(0 \leq i \leq n-1)
$$

where $[D, U]$ is a support of the risky asset price ratio distribution and the notation $C R R_{i}(f, \cdot, \cdot)$ is given in Definition 3.1. The lower bound at time $t_{i}$ of the no-arbitrage price interval does not depend on parameters $D$ and $U$ and is given by the following formula:

$$
\underline{Y}_{i}(f, D, U) \equiv \underline{Y}_{i}(f)=(1+r)^{-(n-i)} f\left(S_{i}(1+r)^{n-i}\right) .
$$

Example 3.4. Suppose $H$ is a European type-option with the pay-off function $f$ that depends only on the terminal risky asset value. Then the upper bound $\bar{Y}_{i}(f, D, U)$ is given by formulas (3.2), (3.3), and (3.4), where $\mathfrak{D}=D$ and $\mathfrak{U}=U$.

Each no-arbitrage price of a contingent claim at time $t_{i}$ is associated with a point within the no-arbitrage price interval $\left(\underline{Y}_{i}(f), \bar{Y}_{i}(f, D, U)\right)(i=0, \ldots, n-1)$. In turn, any point within this interval can be explicitly expressed in terms of the additional model parameters $d$ and $u(D<d<(1+r)<u<U)$. We have the following proposition which follows from the convexity assumption (A2) (see, e.g., [20]).

Proposition 3.5. Suppose assumptions (A1) and (A2) hold. Let $y_{i}$ be an arbitrary point within the no-arbitrage price interval $\left(\underline{Y}_{i}(f), \bar{Y}_{i}(f, D, U)\right)(i=0, \ldots, n-1)$.

Then there exists at least one pair of numbers $(d, u)(D<d<(1+r)<u<U)$ such that $y_{i}$ can be represented in the following form:

$$
y_{i}=C R R_{i}(f, d, u),
$$

where the notation $C R R_{i}(f, \cdot, \cdot)$ is explained in Definition 3.1.

Example 3.6. In the case where $H$ is a European-type path-independent option with the pay-off function $f$, an arbitrary point within the no-arbitrage price interval $\left(\underline{Y}_{i}(f), \bar{Y}_{i}(f, D\right.$, $\mathcal{U}))(i=0, \ldots, n-1)$ is given by formulas (3.2), (3.3), and (3.4), where $\mathfrak{D}=d$ and $\mathfrak{U}=u$. 
Remark 3.7. For each point $y_{i}$ within the no-arbitrage price interval

$$
\left(\underline{Y}_{i}(f), \bar{Y}_{i}(f, D, U)\right),
$$

there is an infinite number of pairs $(d, u)$ such that (3.7) is satisfied.

\subsection{Optimal Hedging}

For the remainder of the paper, we will focus on the case where a convex payoff function $f$ of a European contingent claim $H$ depends on the terminal risky asset value, but not on the risky asset price path. The case of a path-dependent $H$ was developed in [20], and this extension is not required for our application to an equity-linked pure endowment insurance contract.

Our goal is to hedge a European contingent claim $H$ with a dynamic hedging portfolio consisting of risky assets and bonds. A hedging portfolio (or, equivalently, a hedging strategy) $\varphi$ is determined by a pair of $F_{i}$-measurable discrete-time stochastic processes $\left(\xi_{i}, \eta_{i}\right)_{i=0,1, \ldots, n-1}$, where $\xi_{i}$ is a number of risky asset units held over the time interval $\left[t_{i}, t_{i+1}\right)$ and $\eta_{i}$ is the number of bonds held over the time interval $\left[t_{i}, t_{i+1}\right)$.

We take a position of a contingent claim seller who possesses an initial capital $C_{0}$ at time $t=0$ and would like to build a hedging strategy consistent with this available initial capital. We will always assume that the amount $C_{0}$ falls within the no-arbitrage contingent claim price interval $\left(\underline{Y}_{0}(f), \bar{Y}_{0}(f, D, U)\right)$ (corresponding to $\left.t=0\right)$. It follows from Proposition 3.5 and Example 3.6 that $C_{0}$ can be calculated as a unique time zero price of the contingent claim $H$ in the framework of the CRR complete binomial market model with parameters $d$ and $u(D<d<(1+r)<u<U)$ :

$$
C_{0}=g_{0}\left(d, u, S_{0}\right)
$$

where $g_{0}$ is given by (3.3) with $i=0, \mathfrak{D}=d$, and $\mathfrak{U}=u$. We recall (see Remark 3.7) that in our incomplete market model there is an infinite number of $(d, u)$ pairs that determine the value $\mathrm{C}_{0}$.

Definition 3.8. Let $C_{0}$ be a point within the interval $\left(\underline{Y}_{0}(f), \bar{Y}_{0}(f, D, U)\right)$. The infinite set of pairs $(d, u)(D<d<(1+r)<u<U)$ that satisfy formula (3.9) is called the admissible parameter set associated with $C_{0}$. We will denote this set by $\Sigma$.

In [16], the idea of utilizing formulas for hedging strategies from the appropriate complete binomial model was first introduced. Further, it was successfully developed in [18-20]. Specifically, A. V. Nagaev and S. A. Nagaev in [16] suggest the following approach to hedging in our incomplete market model. For a given initial capital $C_{0}$ and for any pair $(d, u)$ in the admissible parameter set $\Sigma$, the hedger uses the amount $C_{0}$ to set up the hedging portfolio $\left(\xi_{0}(d, u), \eta_{0}(d, u)\right)$. This portfolio will be rebalanced at every hedging time $t_{i}, i=1, \ldots, n-1$ according to the following formulas:

$$
\begin{gathered}
\xi_{i}(d, u)=\frac{g_{i+1}\left(d, u, S_{i} u\right)-g_{i+1}\left(d, u, S_{i} d\right)}{S_{i}(u-d)} \\
\eta_{i}(d, u)=\frac{u g_{i+1}\left(d, u, S_{i} d\right)-d g_{i+1}\left(d, u, S_{i} u\right)}{(1+r) B_{i}(u-d)}
\end{gathered}
$$

(here $g_{i}$ is given by (3.3) with $\mathfrak{D}=d$ and $\mathfrak{U}=u$ ) creating a hedging strategy $\varphi(d, u)$. 
In the framework of the CRR complete binomial model with parameters $d$ and $u$, formulas (3.10) define a unique self-financing hedging strategy that perfectly replicates the contingent claim $H$. The same formulas play a different role in the framework of the present incomplete model. It follows from Remark 3.7 that for each initial capital $C_{0}$ there is an infinite set of hedging strategies $\varphi(d, u)$ given by formulas (3.10) that are parameterized by the pairs $(d, u)$ in the admissible parameter set $\Sigma$. Moreover, these strategies are non-self-financing, as will be now explained.

Let us fix a $(d, u)$-pair in $\Sigma$ and consider the associated hedging strategy (3.10) $(i=$ $0, \ldots, n-1)$. It was shown in [18] that at every hedging time $t_{i}(i=1, \ldots, n)$ the hedging strategy produces a nonzero local residual amount $\delta_{i}$, a difference between the liquidation value at time $t_{i}$ of the time $t_{i-1}$ hedging portfolio and the set-up cost of the hedging portfolio held from $t_{i}$ to $t_{i+1}$. The local residual amount depends on the parameters $(d, u)$ and has the following explicit form:

$$
\delta_{i}(d, u)=\frac{u-\psi_{i}}{u-d} g_{i}\left(d, u, S_{i-1} d\right)+\frac{\psi_{i}-d}{u-d} g_{i}\left(d, u, S_{i-1} u\right)-g_{i}\left(d, u, S_{i-1} \psi_{i}\right), \quad i=1, \ldots, n
$$

(we recall that $\psi_{i}=S_{i} / S_{i-1}$ is a risky asset price ratio at time $t_{i}$ ).

The local residual amounts $\delta_{i}(d, u)$ are in general nonzero. More precisely, the sign of $\delta_{i}(d, u)$ depends on the risky asset price ratio $\psi_{i}$ :

(i) $\delta_{i}(d, u)>0$ if $d<\psi_{i}<u$,

(ii) $\delta_{i}(d, u)=0$ if $\psi_{i}=d$ or $\psi_{i}=u$,

(iii) $\delta_{i}(d, u)<0$ if $D<\psi_{i}<d$ or $u<\psi_{i}<U$.

The nonzero local residuals imply the fact that for each $(d, u)$ pair in $\Sigma$, formulas (3.10) define a non-self-financing hedging strategy $\varphi(d, u)$ that is created starting with the initial capital $C_{0}$. In order to maintain the hedging strategy $\varphi(d, u)$, at each time step $i=1, \ldots, n$, the investor will either withdraw the local residual $\delta_{i}(d, u)$ from the liquidated proceeds when $\delta_{i}(d, u)$ is positive or add the amount when $\delta_{i}(d, u)$ is negative. Let us notice that such portfolio adjustment made at maturity time $t_{n}=T$ guarantees that the hedging portfolio value at termination time matches the liability $H$. follows:

Let us define the discounted local residual amount at time $t_{i}$ denoted by $\widetilde{\delta}_{i}(d, u)$ as

$$
\widetilde{\delta}_{i}(d, u)=\delta_{i}(d, u)(1+r)^{-i}, \quad i=1, \ldots, n .
$$

The discounted local residuals $\widetilde{\delta}_{i}(d, u)$ produce the accumulated residual amount $\Delta_{n}(d, u)$ :

$$
\Delta_{n}(d, u)=\widetilde{\delta}_{1}(d, u)+\widetilde{\delta}_{2}(d, u)+\cdots+\widetilde{\delta}_{n}(d, u)
$$

In order to choose the "best" hedging strategy out of the infinite set of admissible hedging strategies, we need to introduce some optimization criteria. There are a variety of optimization criteria that are meaningful to the investor and could be expressed in terms of local residuals as well as the accumulated residual amount. We introduce two criteria that will be used in this paper in Section 3.3. 
Since the residuals can be explicitly expressed in terms of the model parameters $(d, u)$ (for a given risky asset price path), the optimization problem involving these criteria can be reduced to the problem of choosing a pair of optimal parameters $\left(d^{*}, u^{*}\right)$ out of the admissible parameter set $\Sigma$. This optimal parameter pair will define an optimal hedging strategy $\varphi\left(d^{*}, u^{*}\right)$.

We develop a numerical optimal hedging algorithm for a variety of contingent claims and underlying assets in [18-20]. Let us notice that the admissible parameter set $\Sigma$ by construction is associated with the given initial capital $C_{0}$. Our algorithm uses the initial hedging capital $C_{0}$ as an input parameter (e.g., $C_{0}$ could be the current market price of the contingent claim). This separates our approach from the approaches of other authors (see, e.g., $[6,7,31])$ who solve a pricing problem together with an optimal hedging problem. One of the advantages of our approach is the possibility for a hedger to use an available initial capital and still achieve optimal hedging results. The only constraint on the initial hedging capital $C_{0}$ is that it must fall within the no-arbitrage price interval described in Section 3.1.

Remark 3.9. The boundary parameters $D, U$ play a purely theoretical role in our setting. As long as one imposes a no-arbitrage assumption on the initial hedging capital $C_{0}$, one can successfully set up and maintain a non-self-financing hedging strategy without knowing the parameters $D$ and $U$.

\subsection{Optimization Criteria and Optimal Hedging Problems}

We will now describe our optimization criteria in more detail. As explained in Section 3.2, local residuals $\delta_{i}(d, u)$ represent actual incremental cash flows in maintaining the hedging strategy. One measure of the risk associated with a particular hedging strategy $\varphi(d, u)$ is based on the outstanding balance of a savings account generated by the cash deposits or loans $\delta_{i}(d, u)$ created by portfolio adjustments at each rebalancing time. The outstanding balance $\mathcal{O}_{i}(d, u)$ in a savings account at time $t_{i}$ associated with the hedging strategy $\varphi(d, u)$ is given by the accumulated local residual amounts to time $i$ :

$$
\mathcal{O}_{i}(d, u)=\sum_{j=1}^{i} \delta_{j}(d, u)(1+r)^{i-j}, \quad i=1, \ldots, n .
$$

Let us notice that $\mathcal{O}_{i}(d, u)$ is a $F_{i}$-measurable random variable for every $i=1, \ldots, n$.

Definition 3.10. The minimum value of the sequence $\left\{\mathcal{O}_{i}\right\}_{i=1}^{n}$ denoted by $\mathcal{M}(d, u)$ will be called the minimum outstanding balance $(\mathrm{MOB})$ associated with the hedging strategy $\varphi(d, u)$ :

$$
\mathcal{M}(d, u)=\min _{1 \leq i \leq n} \mathcal{O}_{i}(d, u)
$$

We notice that $\mathcal{M}(d, u)$ is a $F_{n}$-measurable random variable associated with the strategy $\varphi(d$, $u)$. The quantity $\mathcal{M}(d, u)$ is the largest outstanding loan (if negative) or the lowest balance amount (if positive) produced by the given strategy.

We consider the following risk optimization problem based on the MOB:

$$
\max _{(d, u) \in \Sigma} E(\mathcal{M}(d, u)),
$$

where the expectation is taken with respect to the physical measure $p$. 
Remark 3.11. The quantity $E(\mathcal{M}(d, u))$ provides a measure of risk associated with the hedging strategy $\varphi(d, u)$. This quantity measures the amount of funds required to maintain the hedging strategy $\varphi(d, u)$ (if negative). A positive value of $E(\mathcal{M}(d, u))$ indicates that the investor does not need to add additional funds to maintain the hedging strategy $\varphi(d, u)$, but rather the strategy produces a positive cash flow. Therefore, as the value of $E(\mathcal{K}(d, u))$ increases, the risk of needing additional incremental funding to maintain the hedging portfolio decreases. The optimal value $E\left(\mathcal{M}\left(d^{*}, u^{*}\right)\right)$ represents the lowest risk value associated with the set of admissible model parameters $\Sigma$, which in turn is determined by the initial hedging capital $C_{0}$.

We will now proceed to the second optimization criterion used in this paper. The return on the investment $C_{0}$ in our incomplete market model can be measured as the expected accumulated residual amount $E\left(\Delta_{n}(d, u)\right)$ produced by a non-self-financing hedging strategy $\varphi(d, u)$ with the initial hedging capital $C_{0}$, where $\Delta_{n}(d, u)$ is defined by (3.13).

We consider the following return optimization problem:

$$
\max _{(d, u) \in \Sigma} E\left(\Delta_{n}(d, u)\right)
$$

where the expectation is taken with respect to the physical measure $p$.

Remark 3.12. Throughout the paper we use the notation $\left(d^{*}, u^{*}\right)$ for the optimal parameter pair with respect to the optimization criterion which is currently under consideration. The optimal value $E\left(\Delta_{n}\left(d^{*}, u^{*}\right)\right)$ represents the largest return value associated with the set of admissible model parameters $\Sigma$, which in turn is determined by the initial hedging capital $C_{0}$.

In our past work, the return characteristic $E\left(\Delta_{n}(d, u)\right)$ has been studied extensively. In [22] we point out the connection between the accumulated residual $\Delta_{n}(d, u)$ produced by a non-self-financing hedging strategy and the cumulative cost of hedge associated with that strategy (see, e.g., $[6,7,31])$. Here we consider this characteristic to illustrate the flexibility of our algorithm.

Remark 3.13. Comparing (3.13) with (3.14), one can easily see that the accumulated residual amount $\Delta_{n}(d, u)$ produced by a hedging strategy $\varphi(d, u)$ is related to the outstanding balance at maturity as follows:

$$
\Delta_{n}(d, u)=\mathcal{O}_{n}(d, u)(1+r)^{-n}
$$

Now we can describe our optimal hedging algorithm in detail.

\subsection{Optimal Hedging Algorithm Design}

The algorithm input parameters are the initial hedging capital $C_{0}$, the contingent claim maturity time $T$ and pay-off function $f$, the initial value of the underlying risky asset $S_{0}$, the risk-free interest rate $r$, the number of hedging times $n$, and the set of historical values of the underlying risky asset.

The algorithm is designed as follows. 


\section{(a) Construct Admissible Parameter Set $\Sigma$}

The admissible parameter set $\Sigma$ (see Definition 3.8) is determined numerically using contour construction software, based on (3.9). We determine a discretized set $\widehat{\Sigma}$ which numerically approximates the admissible parameter set $\Sigma$ and consists of a finite number of $(d, u)$ pairs.

\section{(b) Simulate Future Paths for Underlying Risky Asset}

The algorithm can easily incorporate various techniques for simulating risky asset paths. In this paper, we consider bootstrap sampling from a set of historical risky asset value jumps. (see Remark 3.14). The risky asset price paths are constructed from the sampled jumps and the given risky asset initial value.

\section{(c) Calculate Local Residuals}

For each $(d, u)$ pair in the discretized admissible parameter set $\widehat{\Sigma}$, and for each simulated path, construct local residual sequence $\delta_{i}(d, u), i=1, \ldots, n$, using (3.11).

\section{(d) Approximate the Optimization Criterion Value for Each $(d, u)$ Pair}

For each $(d, u)$ pair in the discretized admissible parameter set $\widehat{\Sigma}$, approximate the value of the optimization criterion by computing an appropriate statistic on the simulated paths.

\section{(e) Determine Numerical Solution of the Optimization Problem}

An approximate solution to the chosen optimization problem is found by choosing the largest (or the smallest) approximating value of the optimization criterion over admissible $(d, u)$ pairs. This value corresponds to the optimal parameter pair $\left(d^{*}, u^{*}\right)$ and therefore the optimal hedging strategy $\varphi\left(d^{*}, u^{*}\right)$.

Our optimal hedging algorithm can be easily adjusted to accommodate any optimization criterion based on local residuals $\delta_{i}(d, u)$ or the accumulated residual $\Delta_{n}(d, u)$ produced by a hedging strategy. In this paper, we will use the two optimization criteria described in Section 3.3.

Using the optimal parameter pair $\left(d^{*}, u^{*}\right)$, it is possible to evaluate additional characteristics of the optimal hedging strategy $\varphi\left(d^{*}, u^{*}\right)$ based on the local residuals $\delta_{i}\left(d^{*}, u^{*}\right), i=$ $1, \ldots, n$, produced by $\varphi\left(d^{*}, u^{*}\right)$, by calculating appropriate statistics on the simulated paths. In the case where risk is chosen as the main criterion, the algorithm calculates the return value associated with $\varphi\left(d^{*}, u^{*}\right)$ as an additional characteristic of the chosen hedging strategy. In the case where return is chosen as the main criterion, the algorithm calculates the risk value associated with $\varphi\left(d^{*}, u^{*}\right)$. This way the user obtains the risk-return profile of the optimal hedging strategy.

Remark 3.14. Bootstrap sampling techniques are used in a wide range of financial applications (see, e.g., [32] and the references therein). The mathematical foundations for bootstrap resampling methods can be found in [33]. In this paper, we make the assumption of independent and identically distributed stock price jumps that justifies our use of bootstrap resampling. Models that account for dependence of the jumps can be easily incorporated into the algorithm and have been studied in previous works of the authors [19]. 


\section{Application to Optimal Hedging and Pricing of Equity-Linked Life Insurance Products}

In this section, we extend our approach developed in Section 3 to optimal hedging and pricing of pure endowment life insurance contracts with guarantee.

An equity-linked pure endowment contract with guarantee is described by its pay-off (benefit) at maturity as follows:

$$
\widehat{H}=\max \left\{S_{T}, k S_{0}\right\} \cdot I_{\{T(x)>T\}} .
$$

Here $S_{t}$ is an underlying risky asset (e.g., stock index) value at time $t, T$ is the contract maturity time, $k(0<k \leq 1)$ is a fixed coefficient equal to a percentage of the initial value of the underlying risky asset that is guaranteed to the living insured at maturity. At maturity, the insured person will receive the largest of the two: the guarantee amount $k S_{0}$ or the terminal value $S_{T}$ of the underlying risky asset (provided he/she is alive at maturity).

Let us take the position of an insurance company that on a certain date is trying to determine a fair price of a pure endowment contract with guarantee with maturity time $T$. Following the approach originating from [1], we rewrite (4.1) as follows:

$$
\widehat{H}=\left(k S_{0}+\max \left\{S_{T}-k S_{0}, 0\right\}\right) \cdot I_{\{T(x)>T\}} .
$$

One can see from (4.2) that the considered life insurance contract contains the embedded European call option on the underlying asset $S$, with maturity time $T$ and strike price $k S_{0}$, with the pay-off function at maturity $f\left(S_{T}\right)=\max \left\{S_{T}-k S_{0}, 0\right\}$.

Following the approach described in Section 2, we arrive at the following formula for a (nonunique) no-arbitrage price ${ }_{T} U_{x}$ of the contract at time zero:

$$
{ }_{T} U_{x}={ }_{T} p_{x} k S_{0} e^{-r T}+{ }_{T} p_{x} \mathcal{C}_{0}(S, T) .
$$

Here $\mathcal{C}_{0}(S, T)$ stands for a no-arbitrage time $t=0$ price of the embedded European call option described above. We recall that this price cannot be uniquely determined in an incomplete financial market. On the other hand, the portion ${ }_{T} p_{x} \mathcal{C}_{0}(S, T)$ of the contract price ${ }_{T} U_{x}$ may be used by the insurance company to hedge against financial risk involved in the equitylinked life insurance contract.

We assume that the insurance company is willing to invest the amount ${ }_{T} p_{x} \mathcal{C}_{0}(S, T)$ in a hedging strategy which optimizes a given criterion, as described in Section 3. The amount ${ }_{T} p_{x} \mathcal{C}_{0}(S, T)$ is interpreted as the initial hedging capital $C_{0}$ :

$$
C_{0}={ }_{T} p_{x} \mathcal{C}_{0}(S, T)
$$

It is important for the insurer to determine a reasonable range of $C_{0}$ values. Intuitively, the larger the initial hedging capital $C_{0}$, the better the quality of the hedging strategy associated with $C_{0}$ and therefore the lower financial risk. On the other hand, the lower the $C_{0}$ values, the lower the contract price ${ }_{T} U_{x}$, the more attractive the contract is to a customer.

As explained in Section 3.1, the amount $C_{0}$ can vary within the open interval of noarbitrage prices for the embedded call option. Therefore theoretically it can be made as low 
as the lower bound of this interval, a highly desirable choice from the customer perspective. The upper bound of the no-arbitrage price interval (as is shown in [18]) represents the initial capital for a minimum cost super hedge. This amount, although highly desirable from the insurer perspective, cannot be considered as a realistic choice.

In our approach, we create a grid of no-arbitrage values for $C_{0}$, where the lowest $C_{0}$ value is the lower bound $\underline{Y}_{0}(f)$ given by (3.6) with $i=0$ and $f\left(S_{T}\right)=\max \left(S_{T}-k S_{0}, 0\right)$. The largest $C_{0}$ value in the grid equals $\alpha B S_{0}(S, T)$, where $B S_{0}(S, T)$ is the time zero Black-Scholes price of the above-described embedded call option and $\alpha$ is a constant that can be determined by the user (empirically, if $\alpha$ is chosen from the interval [1,1.2], one obtains a reasonable range of $C_{0}$ values).

Each calculated $C_{0}$ value in the grid is used as an input parameter (initial hedging capital) for the optimal hedging algorithm described in Section 3.4. For each $C_{0}$ value, based on the chosen optimization criterion, this algorithm determines an optimal pair of model parameters $\left(d^{*}, u^{*}\right)$ within the admissible parameter set $\Sigma$ and calculates the optimal hedging strategy $\varphi\left(d^{*}, u^{*}\right)$. The algorithm also calculates the optimal value of the chosen optimization criterion and provides the risk-return profile of the optimal strategy.

Analyzing risk-return profiles of the optimal hedging strategies, the insurance company chooses the $C_{0}$ value which delivers the most acceptable results based on their riskreturn preferences. Once the $C_{0}$ value is chosen, the insurer is able to price pure endowment contracts with guarantee for their customers.

Using (4.4), let us rewrite formula (4.3) as follows:

$$
{ }_{T} U_{x}={ }_{T} p_{x} k S_{0} e^{-r T}+C_{0} .
$$

Based on the customer's age, the survival probability ${ }_{T} p_{x}$ is determined using the available mortality data (see, e.g., [34]) and the contract price ${ }_{T} U_{x}$ is calculated using (4.5) with the chosen $C_{0}$ value.

\section{Illustrative Numerical Results}

We have applied our method described in Section 4 to the optimal hedging and pricing of pure endowment life insurance contracts with guarantee, using the S\&P 500 Index as the underlying risky asset. In this section, we give a description of the numerical results of our simulations.

\subsection{Simulations for Risk Optimization}

We take a position of an insurer who on March 19, 2010, needs to price and hedge two hypothetical pure endowment life insurance contracts with guarantee. The first contract matures in 10 years $(T=10)$, and the second contract matures in 20 years $(T=20)$. In both cases the underlying risky asset is the S\&P 500 Index and the starting date is March 19, 2010. The S\&P 500 Index starting value is $1159.90\left(S_{0}=1159.9\right)$. The guarantee amount in each contract equals $100 \%$ of the risky asset value as of the starting date of the contract $(k=1)$.

We consider two risk-free interest rate $r$ values: $2 \%$ and $4 \%$. For each combination of parameters $(r, T)$, three or four initial hedging capital $C_{0}$ values are computed as described in Section 4 (we use the value $\alpha=1.1$ ). These values are presented in Table 1, column "Hedging capital $C_{0}{ }^{\prime \prime}$. 
Table 1: Illustrative numerical results for two pure endowment contracts with guarantee.

\begin{tabular}{lcccccc}
\hline Case & Rate & Maturity & $\begin{array}{c}\text { Hedging capital } \\
\mathbf{C}_{0}\end{array}$ & $\begin{array}{c}\text { Risk } \\
\mathbf{M}^{*}\end{array}$ & Risk interval & $\begin{array}{c}\text { Return } \\
\overline{\boldsymbol{\Delta}}\end{array}$ \\
\hline 1 & $\boldsymbol{T}$ & $T$ & 206.94 & -1.83 & $(-4.25,2.96)$ & 43.22 \\
2 & 0.02 & 10 & 258.67 & 2.53 & $(0.32,5.49)$ & 78.88 \\
3 & 0.02 & 10 & 323.34 & 4.62 & $(3.18,7.33)$ & 124.92 \\
4 & 0.02 & 10 & 355.67 & 5.43 & $(3.32,7.79)$ & 153.06 \\
5 & 0.02 & 10 & 378.32 & 1.84 & $(0.58,3.95)$ & 114.62 \\
6 & 0.02 & 20 & 442.19 & 2.61 & $(1.17,4.69)$ & 161.54 \\
7 & 0.02 & 20 & 491.32 & 3.15 & $(2.23,4.92)$ & 191.72 \\
8 & 0.02 & 20 & 540.45 & 3.51 & $(2.49,5.04)$ & 222.07 \\
9 & 0.02 & 20 & 373.38 & 6.15 & $(3.38,8.92)$ & 195.05 \\
10 & 0.04 & 10 & 434.16 & 7.18 & $(4.79,10.76)$ & 234.51 \\
11 & 0.04 & 10 & 477.58 & 7.86 & $(5.38,11.07)$ & 256.19 \\
12 & 0.04 & 10 & 629.13 & 3.44 & $(3.04,4.40)$ & 234.49 \\
13 & 0.04 & 20 & 669.29 & 3.54 & $(3.09,4.86)$ & 256.75 \\
14 & 0.04 & 20 & 736.22 & 3.69 & $(3.49,4.93)$ & 288.23 \\
\hline
\end{tabular}

Each initial hedging capital $C_{0}$ is used as an input parameter for the optimal hedging algorithm (see Section 3.4). In this simulation, we numerically solve the theoretical optimization problem (3.16) choosing the expected minimum outstanding balance as the optimization criterion.

In step (b) of the hedging algorithm, two hundred representative S\&P 500 Index value paths are simulated. In this study, we use bootstrap sampling from a set of historical quarterly index value jumps. Our hedging strategies, computed using (3.10), are rebalanced quarterly.

Having calculated local residuals in step (c) of the algorithm, we need to approximate the expected $\mathrm{MOB}$ value in step $(\mathrm{d})$. For each $(d, u)$ pair in the discretized admissible parameter set $\widehat{\Sigma}$, and for each simulated path, we use (3.15) to compute the values $\mathcal{M}(d, u)$. These values are averaged over the simulated paths. The average value (denoted by $\mathbf{M}_{(d, u)}$ ) numerically approximates the expectation $E(\mathcal{M}(d, u))$ :

$$
\mathbf{M}_{(d, u)} \approx E(\mathcal{M}(d, u)) .
$$

In step (e), we determine an approximate solution $\mathbf{M}^{*}$ to the optimization problem (3.16):

$$
\mathbf{M}^{*} \approx \max _{(d, u) \in \Sigma} E(\mathcal{M}(d, u))
$$

To do so, we choose the largest approximating value $\mathbf{M}_{(d, u)}$ over admissible $(d, u)$ pairs:

$$
\mathbf{M}^{*}=\max _{(d, u) \in \hat{\Sigma}} \mathbf{M}_{(d, u)} .
$$

This value corresponds to the optimal parameter pair $\left(d^{*}, u^{*}\right)$ and therefore the optimal hedging strategy $\varphi\left(d^{*}, u^{*}\right)$. 
As an additional characteristic of the optimal hedging strategy $\varphi\left(d^{*}, u^{*}\right)$, we determine an approximate return (denoted by $\overline{\boldsymbol{\Delta}}$ ) associated with the optimal hedging strategy $\varphi\left(d^{*}, u^{*}\right)$ :

$$
\bar{\Delta} \approx E\left(\Delta_{n}\left(d^{*}, u^{*}\right)\right) .
$$

For the optimal pair $\left(d^{*}, u^{*}\right)$, we calculate the accumulated residual $\Delta_{n}\left(d^{*}, u^{*}\right)$ given by (3.13) on each simulated path. An approximate return $\bar{\Delta}$ is found by averaging the accumulated residual values over the simulated paths.

The pair $\left(\mathbf{M}^{*}, \overline{\mathbf{\Delta}}\right)$ describes the risk-return profile of the chosen optimal hedging strategy.

\subsection{Numerical Results for Risk Optimization}

Numerical results of our simulations described in Section 5.1 are presented in Table 1.

For each set of input parameters, in column "Risk" we report the value of $\mathbf{M}^{*}$ (see (5.2)). Let us consider risk values $\mathbf{M}^{*}$ that differ only by the amount of the initial hedging capital $C_{0}$ with the rest of the input parameters fixed. For example, in cases 1 through 4 the initial hedging capital $C_{0}$ increases from 206.94 to 355.67 while $r$ and $T$ are unchanged. As the initial hedging capital increases, the values of $\mathbf{M}^{*}$ also increase from -1.83 to 5.43 , respectively. Larger $\mathbf{M}^{*}$ values correspond to lower risk for the investor (see Remark 3.11 for more detail).

A nonparametric estimator of the confidence interval for $\mathbf{M}^{*}$ is presented in column "Risk Interval." The left endpoint of the interval is the estimate of the lower quartile of the minimum outstanding balance $\mathcal{M}\left(d^{*}, u^{*}\right)$ values (corresponding to the optimal parameter pair $\left.\left(d^{*}, u^{*}\right)\right)$. The right endpoint is the estimate of the upper quartile of $\mathcal{M}\left(d^{*}, u^{*}\right)$ values. It is interesting to note that even the lower quartile value for $\mathcal{M}\left(d^{*}, u^{*}\right)$ is positive in most of the cases presented.

In column "Return" we report the approximate expected accumulated residual $\bar{\Delta}$ (see (5.4)) associated with each optimal hedging strategy $\varphi\left(d^{*}, u^{*}\right)$. Let us return to cases 1 through 4 discussed above. As the risk associated with the hedging strategy decreases $\left(\mathbf{M}^{*}\right.$ values increase from -1.83 to 5.43$)$, the return from the strategy increases ( $\bar{\Delta}$ values increase from 43.22 to 153.06 ).

Figure 1 illustrates the behavior of $\mathbf{M}_{(d, u)}$ (see (5.1)) as $(d, u)$ pairs vary over the admissible parameter set $\widehat{\Sigma}$ for each case presented in Table 1 . The vertical axis of the figure is marked with the case numbers. Each set of circles (located on horizontal lines) represents the set of all possible values of the optimization criterion $\mathbf{M}_{(d, u)}$ produced by the hedging strategies $\varphi(d, u)$, when $(d, u)$ pairs change within the appropriate parameter set $\widehat{\Sigma}$. In case 1 at the bottom of the figure, all $\mathbf{M}_{(d, u)}$ values are negative, but the largest value of -1.83 selected by the algorithm is a vast improvement over the lowest possible value of approximately -20 . Application of the algorithm in case 2 produces similar results with $\mathbf{M}_{\left(d^{*}, u^{*}\right)}=\mathbf{M}^{*}=2.53$ as compared to the lowest possible value of $\mathbf{M}_{(d, u)}$ of approximately -12 . The values of $\mathbf{M}_{(d, u)}$ are not as widely spread in cases 14,13 , and 12 , but in all cases the algorithm produces a positive optimal value of $\mathbf{M}_{(d, u)}$ as compared to a slightly negative possible value without the optimization.

On the basis of the simulation results presented in Table 1, the insurance company can price pure endowment life insurance contracts with guarantee in accordance with their riskreturn preferences. Suppose the insurance company would like to price a 10-year contract assuming the risk-free interest rate of $2 \%$. Suppose, analyzing cases 1 through 4 in Table 1 , 


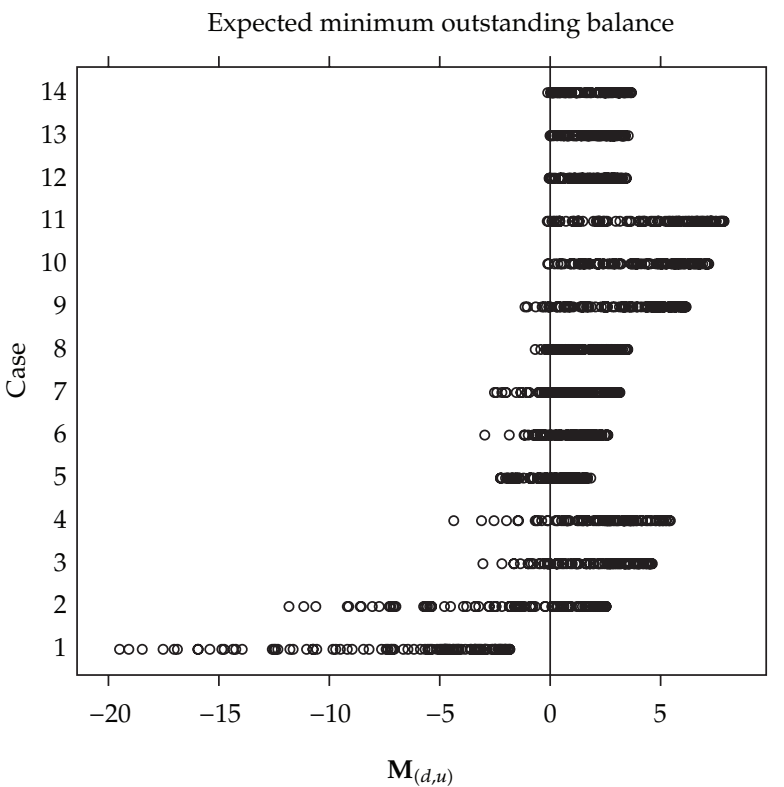

Figure 1: Range of approximate expected minimum outstanding balance $\mathbf{M}_{(d, u)}$ values for all cases.

they find that the risk-return profile of the optimal hedging strategy in case 3 is satisfactory. The corresponding value of the initial hedging capital is 323.34 .

Suppose a 50 -year-old person is interested in purchasing a 10-year life insurance contract. The survival probability ${ }_{10} p_{50}$ is determined using [34]: ${ }_{10} p_{50}=0.9338$. Using (4.5) with $T=10, x=50, r=0.02, k=1, S_{0}=1159.90$, and $C_{0}=323.34$, the insurer calculates the contract price: ${ }_{10} U_{50}=1214.90$. The same contract will cost a 60 -year-old person 1147.21 since the survival probability in this case is only ${ }_{10} p_{60}=0.8676$. A 40 -year-old person will have to pay 1245.52 for the same contract since his/her survival probability is ${ }_{10} p_{40}=0.9711$.

If the insurance company needs to price a 20 -year contract assuming the risk-free interest rate of $2 \%$, they will analyze cases 5 through 8 . Suppose the risk-return profile of the optimal hedging strategy in case 6 is satisfactory. The corresponding initial hedging capital is 442.19.

Reasoning along similar lines as in the previous case, the insurance company prices the 20-year life insurance contracts for 40-, 50-, and 60-year-old customers. The contract prices are $1151.02,1075.46$, and 913.63, respectively.

\subsection{Simulations for Return Optimization}

Let us consider the same two hypothetical life insurance contracts described before in Section 5.1. With the same set of input parameters and the same initial hedging capitals (cases 1 through 14 in Table 1), we will apply our optimal hedging algorithm described in Section 3.4, where the expected accumulated residual produced by a hedging strategy is chosen as the main optimization criterion.

Steps (a) through (c) of the algorithm are the same as in Section 5.1. In step (d), the expected accumulated residual amount $E\left(\Delta_{n}(d, u)\right)$ is approximated. For each $(d, u)$ pair in the discretized admissible parameter set $\widehat{\Sigma}$, and for each simulated path, we compute 
the accumulated residual $\Delta_{n}(d, u)$ using (3.13). For each $(d, u)$ pair in $\widehat{\Sigma}$, the accumulated residual $\Delta_{n}(d, u)$ is averaged over the simulated paths. This average value (denoted by $\left.\boldsymbol{\Delta}_{(d, u)}\right)$ numerically approximates the expectation $E\left(\Delta_{n}(d, u)\right)$ :

$$
\Delta_{(d, u)} \approx E\left(\Delta_{n}(d, u)\right)
$$
(3.17):

In step (e), we determine an approximate solution $\Delta^{*}$ to the optimization problem

$$
\Delta^{*} \approx \max _{(d, u) \in \Sigma} E\left(\Delta_{n}(d, u)\right)
$$

The value $\boldsymbol{\Delta}^{*}$ is the largest approximating value $\boldsymbol{\Delta}_{(d, u)}$ over admissible $(d, u)$ pairs:

$$
\Delta^{*}=\max _{(d, u) \in \widehat{\Sigma}} \Delta_{(d, u)}
$$

This value corresponds to the optimal parameter pair $\left(d^{*}, u^{*}\right)$ and therefore the optimal hedging strategy $\varphi\left(d^{*}, u^{*}\right)$.

As an additional characteristic of the optimal hedging strategy $\varphi\left(d^{*}, u^{*}\right)$, we determine an approximate risk (denoted by $\overline{\mathbf{M}}$ ) associated with $\varphi\left(d^{*}, u^{*}\right)$ :

$$
\overline{\mathbf{M}}=\mathbf{M}_{\left(d^{*}, u^{*}\right)},
$$

where $\mathbf{M}_{\left(d^{*}, u^{*}\right)}$ is the numerical approximation of the expectation $E\left(\mathcal{M}\left(d^{*}, u^{*}\right)\right)$ (see (5.1)). For the optimal pair $\left(d^{*}, u^{*}\right)$, we calculate the minimum outstanding balance $\mathcal{M}\left(d^{*}, u^{*}\right)$ given by (3.15) on each simulated path. The approximate risk $\overline{\mathbf{M}}$ is found by averaging $\mathcal{M}\left(d^{*}, u^{*}\right)$ over the simulated paths.

The pair $\left(\Delta^{*}, \overline{\mathbf{M}}\right)$ will describe the risk-return profile of the chosen optimal hedging strategy.

\subsection{Numerical Results for Return Optimization}

Numerical results for applying the algorithm with the alternative optimization criterion are generated for each of the fourteen cases described in Table 1 and are presented in Table 2.

In column "Return" we report the optimal value of the expected accumulated residual $\Delta^{*}$. The "Risk" column presents the corresponding risk value $\overline{\mathbf{M}}$ associated with the optimal hedging strategy $\varphi\left(d^{*}, u^{*}\right)$.

Analogous to Figure 1 that depicts the behavior of the risk $\mathbf{M}_{(d, u)}$, Figure 2 illustrates the behavior of the return $\boldsymbol{\Delta}_{(d, u)}$ (see (5.5)) as $(d, u)$ pairs vary over the admissible parameter set $\widehat{\Sigma}$ for each case presented in Table 1 .

Comparing the results presented in Table 2 with those presented in Table 1, we can see the risk versus return tradeoff. Since the return characteristic is optimized in results contained in Table 2, the value of $\boldsymbol{\Delta}^{*}$ in Table 2 is larger than the value of $\bar{\Delta}$ presented in Table 1 for each case. The optimal return can be as much as $20 \%$ larger than the value reported in Table 1 , as seen in case 3 , or as small as $0.5 \%$ larger, as seen in case 12 . 
Table 2: Risk-return profile for return optimization.

\begin{tabular}{lcc}
\hline Case & Return & Risk \\
& $\Delta^{*}$ & $\overline{\mathbf{M}}$ \\
\hline 1 & 51.872 & -4.654 \\
2 & 94.611 & 0.762 \\
3 & 161.106 & -0.438 \\
4 & 160.947 & 2.845 \\
5 & 128.366 & 0.978 \\
6 & 165.268 & 1.767 \\
7 & 194.585 & 2.437 \\
8 & 229.673 & 3.489 \\
9 & 199.127 & 6.094 \\
10 & 241.652 & 4.420 \\
11 & 263.344 & 7.773 \\
12 & 235.642 & 2.705 \\
13 & 260.138 & 2.893 \\
14 & 291.412 & 2.645 \\
\hline
\end{tabular}

Figure 3 depicts two return values for each of the 14 considered cases: the optimal return $\Delta^{*}$ (marked with the bold dot) and the return value $\bar{\Delta}$ associated with the optimal risk (marked with the unfilled dot).

While the return is larger in each case, the risk $\overline{\mathbf{M}}$ is also larger. Compare the value $\mathbf{M}^{*}=-1.83$ in case 1 , in Table 1, to $\overline{\mathbf{M}}=-4.654$, in Table 2. Each value represents the numerical approximation of the expected minimum outstanding balance produced by the corresponding hedging strategy. The larger negative value of this characteristic indicates the larger risk. For this particular case, we can see that while the return associated with the hedging strategy that optimizes return is approximately $20 \%$ larger, the risk is more than $250 \%$ larger. Similarly, in case $3, \mathbf{M}^{*}=4.62$ in Table 1, where risk is optimized. The corresponding risk value in Table 2 is $\overline{\mathbf{M}}=-0.438$ which indicates a larger risk involved in producing the improved return.

In Figure 4, two risk values are presented for each of the 14 cases: the optimal risk $\mathbf{M}^{*}$ (marked with the bold dot) and the risk value $\overline{\mathbf{M}}$ associated with the optimal return (marked with the unfilled dot).

In summary, the results in Tables 1 and 2 provide an interesting comparison between risk and return. Since the algorithm can optimize either risk or return, the user is able to determine the criterion that is most relevant. Examining results of applying the algorithm with each optimization criterion provides a method for evaluating the risk versus return trade-off.

Pricing of pure endowment contracts with guarantee on the basis of the return optimization will require analyzing the results presented in Table 2. Having chosen the satisfactory risk-return profile of the optimal hedging strategy, the insurer identifies the corresponding hedging capital. Then the survival probability of an insured person is determined and the contract price is calculated using (4.5).

\section{Hedging Strategy Recalibration}

In contrast to classical equity option valuations, which typically arise over a short time horizon (e.g., 6 months to 2 years), the time to expiration of a financial option embedded in 


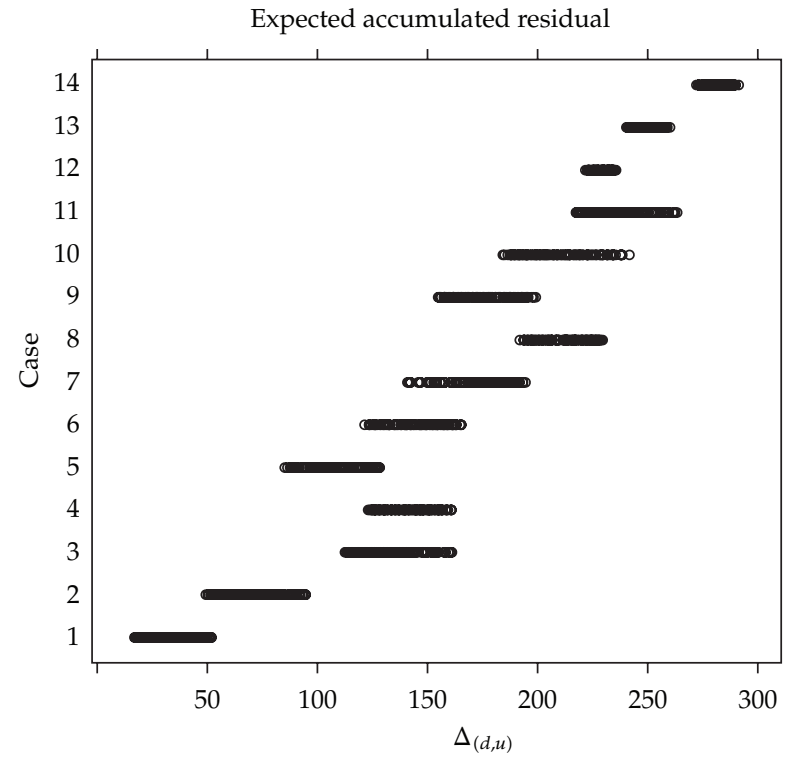

Figure 2: Range of approximate expected accumulated residual $\boldsymbol{\Delta}_{(d, u)}$ values for all cases.

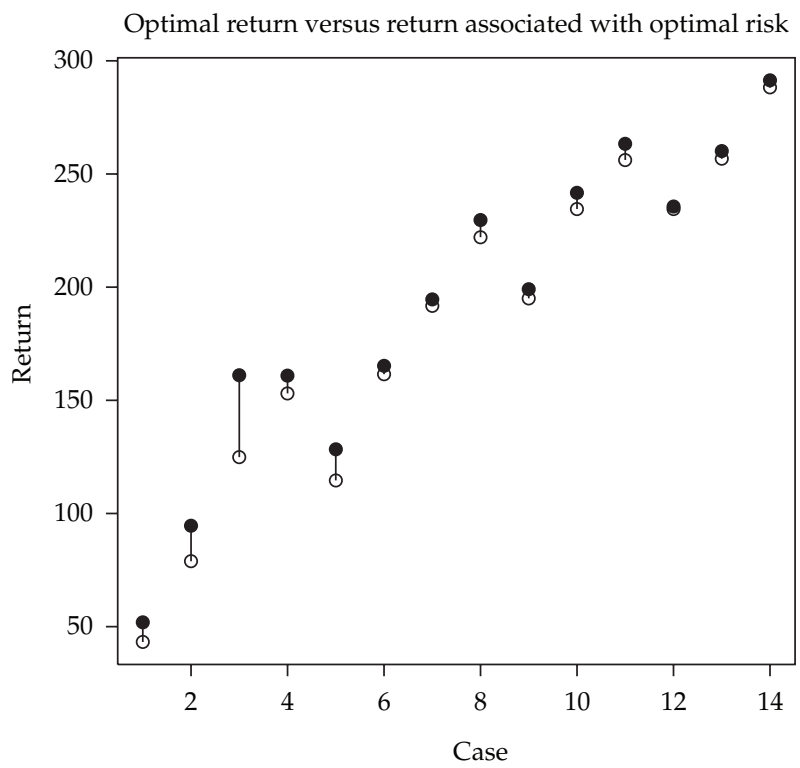

Figure 3: Optimal return $\Delta^{*}$ versus the return value $\bar{\Delta}$ associated with the optimal risk for all cases.

an equity-linked insurance contract can be 10 years or longer. This raises the possibility that the financial market conditions may change significantly over the lifetime of the insurance contract. Since model parameters are determined using historical asset value paths, these original parameter values may not accurately reflect market conditions as the contract matures. A benefit of our algorithmic approach to hedging a financial risk embedded in a long-term equity-linked life insurance contract is the ability to recalibrate the model 


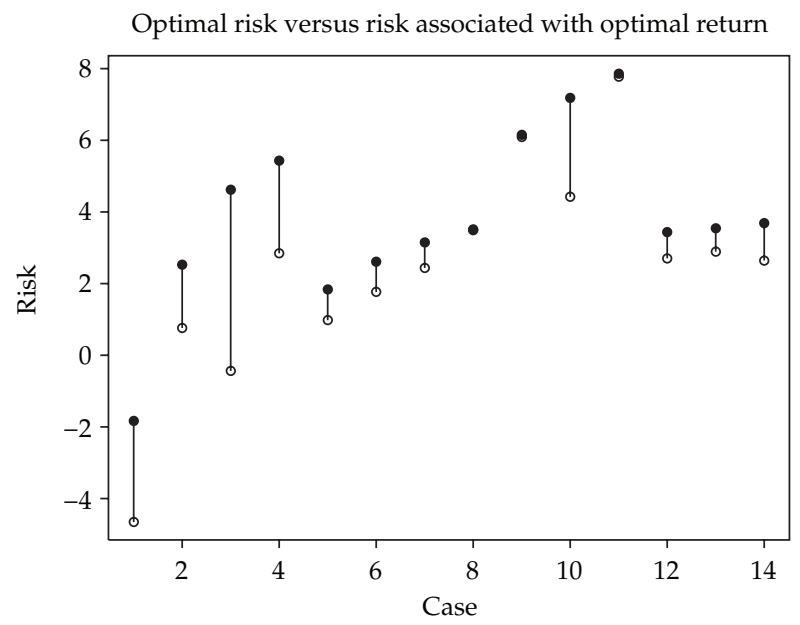

Figure 4: Optimal risk $\mathbf{M}^{*}$ versus the risk value $\overline{\mathbf{M}}$ associated with the optimal return for all cases.

parameters at any point during the contact period using the latest financial market data. In this section, we illustrate the flexibility of our algorithm by using the most recent market data to adjust the algorithm input parameters to more accurately reflect current market conditions.

Consider a hypothetical long-term European call option embedded in an equity-linked life insurance contract initiated on March 19, 2000, and maturing on March 19, 2010. Let us emphasize that we use the past time period for this set of numerical experiments so that the historical market data is available.

The underlying risky asset is the S\&P 500 index, and the value on the starting date is $S_{0}=1456.63$. The call option is at the money as of the starting date of the contract (the strike price equals $S_{0}$ ), and the maturity time is $T=10$. The available initial hedging capital is $C_{0}=406.55$.

Let us take the position of the option seller who on March 19, 2000, would like to determine a return-optimal hedging strategy for the lifetime of the option. The hedging strategy will be rebalanced quarterly.

We will explore two possible approaches to this problem. The first approach consists of using the return maximization hedging algorithm to determine an optimal hedging strategy for the whole lifetime of the option (a ten year period 2000-2010). We will refer to this hedging strategy as the "original" strategy.

The second approach consists of using the original hedging strategy only for the first five years of the option contract life (2000-2005) and then switching to an updated hedging strategy that takes into account the latest market data for the remaining lifetime of the option (2005-2010). We will refer to this hedging strategy as the "Recalibrated" strategy.

The two strategies are evaluated by comparing the size of the return produced by each strategy. In order to do so, we will use the actual S\&P 500 Index data available for 2000-2010.

In order to determine the original hedging strategy, we use the return optimization hedging algorithm with $C_{0}=406.55$. The S\&P 500 Index values for the ten-year historical period 1990-2000 are used as input for simulation of the "future" risky asset paths. The value of the risk-free interest rate is fixed at $r=0.02$. The original optimal hedging strategy $\varphi\left(d^{*}, u^{*}\right)$ (associated with the optimal parameter pair $\left(d^{*}, u^{*}\right)$ ) is determined. This strategy is then applied to the actual S\&P 500 Index path for 2000-2010 and the corresponding return 
Table 3: Return values for original and recalibrated strategies (actual S\&P 500 Index path for 2005-2010).

\begin{tabular}{lr}
\hline & Return \\
\hline Original strategy & 128.15 \\
Recalibrated strategy & 208.92 \\
\hline
\end{tabular}

Table 4: Return statistics for original and recalibrated strategies (200 simulated S\&P 500 Index paths for 2005-2010).

\begin{tabular}{lccc}
\hline \multicolumn{1}{c}{ Original strategy } & & \multicolumn{2}{c}{ Recalibrated strategy } \\
\hline 1-4 Min & -99.66 & Min & -107.07 \\
1st Quarter & 22.27 & 1st Quarter & 95.79 \\
Median & 60.93 & Median & 120.23 \\
Mean & 59.22 & Mean & 115.71 \\
3rd Quarter & 97.843 & 3rd Quarter & 151.23 \\
Max & 186.65 & Max & 254.15 \\
\hline
\end{tabular}

$\Delta_{n}\left(d^{*}, u^{*}\right)$ is calculated. We will be interested only in the part of the return accumulated over the last five years of the contract (2005-2010). This accumulated return equals 128.15 (see Table 3).

The recalibrated strategy is identical to the original optimal strategy for the first five years (2000-2005). After five years, the strategy is terminated and the existing hedging portfolio is liquidated. The amount of new hedging capital available for the rest of the option life (2005-2010) is determined. It consists of the liquidation value of the hedging portfolio at the end of five years combined with the outstanding balance (3.14) (as of the end of five years) in the savings account used to accumulate local residual cash flows.

The optimal hedging algorithm is used again on March 19, 2005, with the new available hedging capital of $C_{0}=374.41$ and the most recent historical values of the S\&P 500 Index (2000-2005) that are used for simulation of the "future" risky asset paths. In this experiment we use the same value of the risk-free interest rate $r=0.02$, although this parameter could be easily changed if needed to reflect the most recent market conditions. The new risky asset starting value is $S_{0}=1189.65$ and the new maturity time is $T=5$. This results in a new hedging strategy $\varphi\left(d_{1}^{*}, u_{1}^{*}\right)$ to be used over the remaining five years of the option. In summary, the recalibrated strategy is the combination of two five-year strategies. The strategy $\varphi\left(d^{*}, u^{*}\right)$ is used over the first five years of the contract, and the strategy $\varphi\left(d_{1}^{*}, u_{1}^{*}\right)$ is used for the last five years of the contract.

The recalibrated strategy is applied to the actual S\&P 500 Index path for 2000-2010 and the corresponding return is calculated. The return accumulated over the last five years of the contract (2005-2010) is reported in Table 3. In this simulation, the recalibrated strategy produces a return that is over one and a half times as large as the return produced by the original strategy.

The results in Table 3 are produced using only a single asset value path, the S\&P 500 Index from 2000 to 2010. A more robust statistical description of the return is presented in Table 4. These results are created using two hundred simulated S\&P 500 Index paths for the period 2005-2010. The paths are simulated by bootstrap sampling from the actual S\&P 500 Index jumps over the 2005-2010 time period. Each of the two hedging strategies (original and recalibrated) are applied to the two hundred simulated paths. A statistical description of the results of these simulations are presented in Table 4 . We note that the recalibrated strategy has 
mean return values nearly twice as large as those of the original strategy. These simulation results reconfirm that the recalibrated strategy is an improvement over the original strategy.

In this numerical experiment, the date of the hedging strategy recalibration was chosen in the middle of the option lifetime. A practitioner may use his/her own judgement as well as available market data to choose the date of the recalibration. The strategy recalibration can be performed as many times as necessary during the option lifetime.

\section{References}

[1] M. J. Brennan and E. S. Schwartz, "The pricing of equity-linked life insurance policies with an asset value guarantee," Journal of Financial Economics, vol. 3, no. 3, pp. 195-213, 1976.

[2] M. Brennan and E. Schwartz, "Alternative investment strategies for the issuer of equity linked life insurance policies with an asset value guarantee," Journal of Business, vol. 52, no. 1, pp. 63-93, 1979.

[3] A. R. Bacinello and F. Ortu, "Pricing equity-linked life insurance with endogenous minimum guarantees," Insurance: Mathematics \& Economics, vol. 12, no. 3, pp. 245-257, 1993.

[4] K. K. Aase and S.-A. Persson, "Pricing of unit-lined life insurance policies," Scandinavian Actuarial Journal, no. 1, pp. 26-52, 1994.

[5] T. Møller, "Hedging equity-linked life insurance contracts," North American Actuarial Journal, vol. 5, no. 2, pp. 79-95, 2001.

[6] H. Föllmer and D. Sondermann, "Hedging of non-redundant contingent claims," in Contributions to Mathematical Economics in Honor of Gerard Debreu, W. Hildebrand and A. Mas-Collel, Eds., pp. 205-223, Elsevier, North-Holland, 1986.

[7] H. Föllmer and M. Schweizer, "Hedging by sequential regression: an introduction to the mathematics of option trading," ASTIN Bulletin, vol. 19, pp. 29-41, 1988.

[8] A. Melnikov and V. Skornyakova, "Quantile hedging and its application to life insurance," Statistics E Decisions, vol. 23, no. 4, pp. 301-316, 2005.

[9] A. V. Melnikov, "Efficient hedging of equity-linked life insurance policies," Doklady Mathematics, vol. 396, no. 6, pp. 752-754, 2004.

[10] A. Melnikov and Y. Romanyuk, "Efficient hedging and pricing of equity-linked life insurance contracts on several risky assets," International Journal of Theoretical and Applied Finance, vol. 11, no. 3, pp. 295-323, 2008.

[11] H. Föllmer and P. Leukert, "Quantile hedging," Finance and Stochastics, vol. 3, no. 3, pp. 251-273, 1999.

[12] H. Föllmer and P. Leukert, "Efficient hedging: cost versus shortfall risk," Finance and Stochastics, vol. 4, no. 2, pp. 117-146, 2000 .

[13] P. Klusik and Z. Palmowski, "Quantile hedging for equity-linked contracts," Insurance: Mathematics $\mathcal{E}$ Economics, vol. 48, no. 2, pp. 280-286, 2011.

[14] S. Jaimungal and V. R. Young, "Pricing equity-linked pure endowments with risky assets that follow Lévy processes," Insurance: Mathematics E Economics, vol. 36, no. 3, pp. 329-346, 2005.

[15] A. Consiglio and D. De Giovanni, "Evaluation of insurance products with guarantee in incomplete markets," Insurance: Mathematics and Economics, vol. 42, no. 1, pp. 332-342, 2008.

[16] A. V. Nagaev and S. A. Nagaev, "Asymptotics of riskless profit under selling of discrete time call options," Applicationes Mathematicae, vol. 30, no. 2, pp. 173-191, 2003.

[17] G. Tessitore and J. Zabczyk, "Pricing options for multinomial models," Polish Academy of Sciences. Bulletin. Mathematics, vol. 44, no. 3, pp. 363-380, 1996.

[18] N. Josephy, L. Kimball, V. Steblovskaya, and A. Nagaev, "An algorithmic approach to non-selffinancing hedging in a discrete-time incomplete market," Discrete Mathematics and Applications, vol. 17, no. 2, pp. 189-207, 2007.

[19] N. Josephy, L. Kimball, and V. Steblovskaya, "A time-series approach to non-self-financing hedging in a discrete-time incomplete market," Journal of Applied Mathematics and Stochastic Analysis, Article ID 275217, 20 pages, 2008.

[20] N. Josephy, L. Kimball, and V. Steblovskaya, “Optimal hedging of path-dependent options in discrete time incomplete market," Communications on Stochastic Analysis, vol. 2, no. 3, pp. 385-404, 2008.

[21] H. Föllmer and A. Schied, Stochastic Finance: An Introduction in Discrete Time, vol. 27, Walter de Gruyter, Berlin, Germany, 2002.

[22] N. Josephy, L. Kimball, and V. Steblovskaya, "Alternative hedging in a discrete-time incomplete market," Journal of Risk. In press. 
[23] J. Cox, S. Ross, and M. Rubinstein, "Option pricing: A simplified approach," Journal of Financial Economics, vol. 7, pp. 229-263, 1979.

[24] E. Luciano and E. Vigna, "Non-mean-reverting affine processes for stochastic mortality," in Proceedings of the 15th International AFIR Colloquium, Zurich, Switzerland, September 2005.

[25] A. V. Melnikov, "Quantile hedging of equity-linked life insurance policies," Doklady mathematics, vol. 96, no. 5, pp. 428-430, 2004.

[26] H. Levy and A. Levy, "Option valuation: an extension of the binomial model," Advances in Futures and Options Research, vol. 5, pp. 46-69, 1991.

[27] M. Schäl, "Martingale measures and hedging for discrete-time financial markets," Mathematics of Operations Research, vol. 24, no. 2, pp. 509-528, 1999.

[28] G. Wolczynska, "An explicit formula for option pricing in discrete incomplete markets," International Journal of Theoretical and Applied Finance, vol. 1, no. 2, pp. 283-288, 1998.

[29] O. Hammarlid, "On minimizing risk in incomplete markets," International Journal of Theoretical and Applied Finance, vol. 1, no. 2, pp. 227-233, 1998.

[30] L. Ruschendorf, "On upper and lower prices in discrete-time models," Proceedings of the Steklov Institute of Mathematics, vol. 237, pp. 134-139, 2002.

[31] T. Coleman, Y. Li, and M.-C. Patron, "Discrete hedging under piecewise linear risk minimization," Journal of Risk, vol. 5, pp. 39-65, 2003.

[32] D. Ruppert, Statistics and Finance: An Introduction, Springer, New York, NY, USA, 2004.

[33] S. N. Lahiri, Resampling Methods for Dependent Data, Springer Series in Statistics, Springer, New York, NY, USA, 2003.

[34] E. Arias, B. Rostron, and B. Tejada-Vera, “United States Life Tables, 2005,” National Vital Statistics Reports, vol. 58, no. 10, pp. 1-40, 2010. 


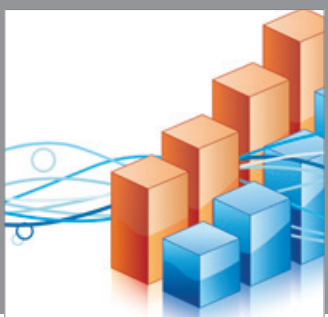

Advances in

Operations Research

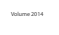

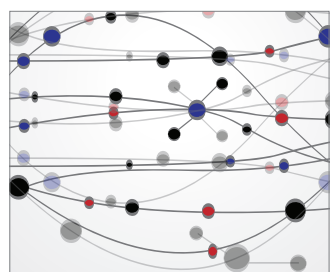

\section{The Scientific} World Journal
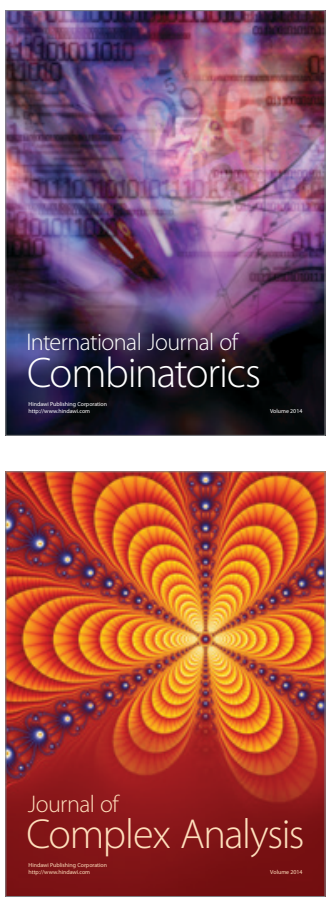

International Journal of

Mathematics and

Mathematical

Sciences
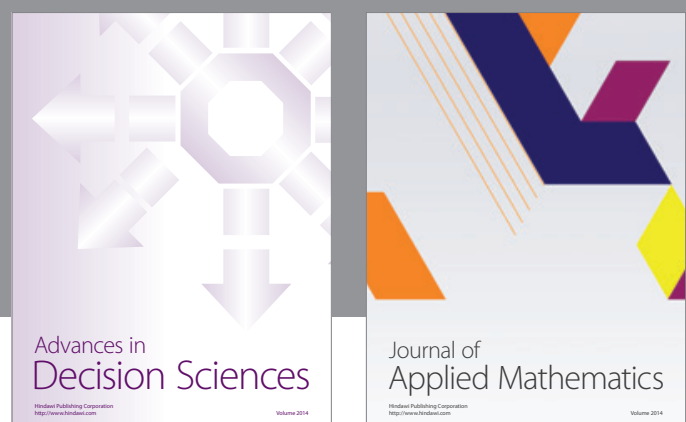

Journal of

Applied Mathematics
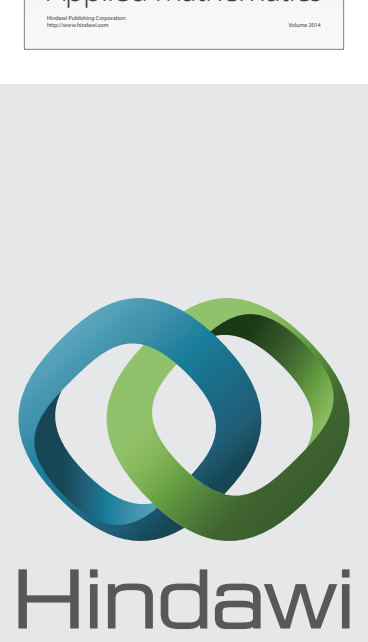

Submit your manuscripts at http://www.hindawi.com
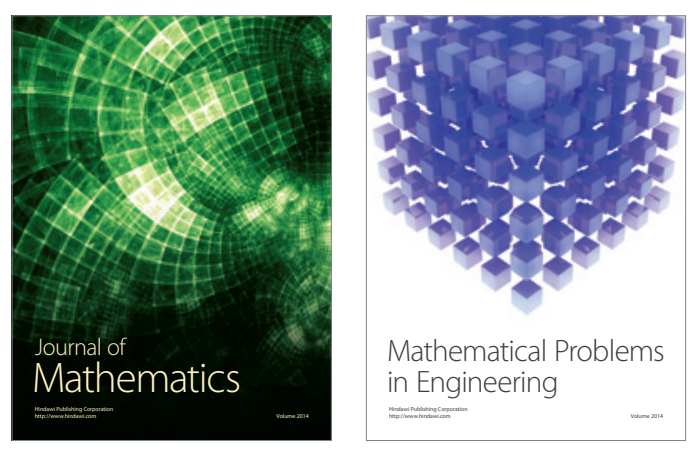

Mathematical Problems in Engineering
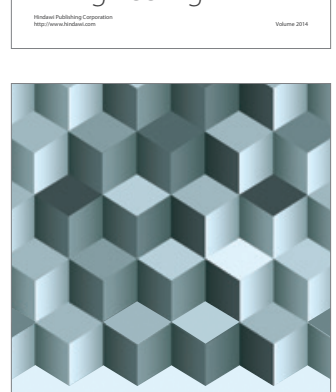

Journal of

Function Spaces
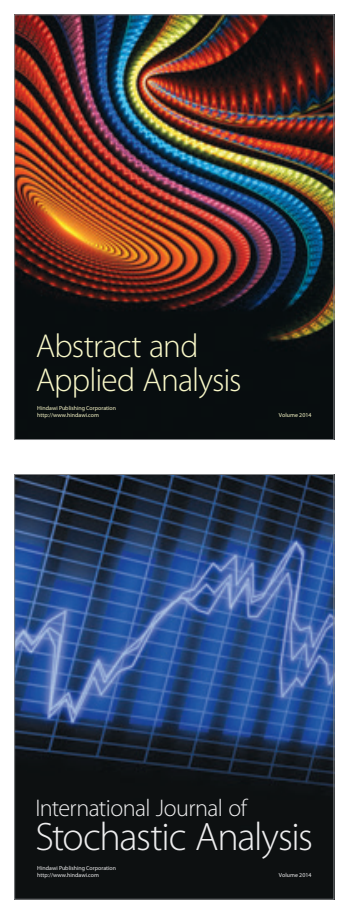

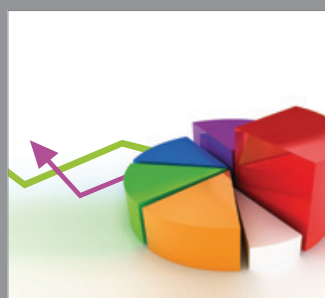

ournal of

Probability and Statistics

Promensencen
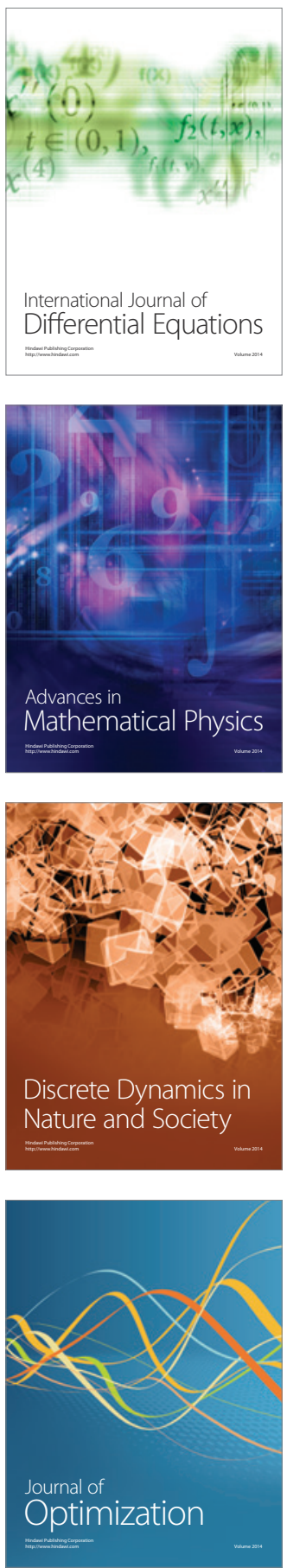